Running head: Gaze discrimination and joint attention

Joint attention facilitates observed gaze direction discrimination

S. Gareth Edwards ${ }^{1}$, Nathalie Seibert ${ }^{2} \&$ Andrew P. Bayliss ${ }^{1}$

1. University of East Anglia

2. Goethe University, Frankfurt

\begin{abstract}
Author Note
S. Gareth Edwards and Andrew P. Bayliss, School of Psychology, University of East Anglia; Nathalie Seibert, Goethe University, Frankfurt.

SGE and APB conceived the idea and designed the experiment. SGE conducted the experiments. SGE \& NS analysed the data. All authors interpreted the data. SGE and APB wrote the manuscript. All authors approved the final manuscript.

This research was supported by a University of East Anglia PhD Studentship to SGE and a Leverhulme Trust Project Grant RPG-2016-173 to APB.

Correspondence concerning this article should be addressed to Andrew P. Bayliss at the School of Psychology, Lawrence Stenhouse Building, University of East Anglia, Norwich, NR4 7TJ, UK. Email: Andrew.P.Bayliss@uea.ac.uk
\end{abstract}




\begin{abstract}
Efficiently judging where someone else is looking is important for social interactions, allowing us a window into their mental state by establishing joint attention. Previous work has shown that judging the gaze direction of a non-foveally-presented face is facilitated when the eyes of that face are directed towards the centre of the scene. This finding has been interpreted as an example of the human bias for misattributing observed ambiguous gaze signals as self-directed eye contact. To test this interpretation against an alternative hypothesis that the facilitation is instead driven by the establishment of joint attention, we conducted two experiments in which we varied the participants' fixation location. In both experiments we replicated the previous finding of facilitated gaze discrimination when the participants fixated centrally. However, this facilitation was abolished when participants fixated peripheral fixation crosses (Experiment 1) and reversed when participants fixated peripheral images of real-world objects (Experiment 2). Based on these data, we propose that the facilitation effect is consistent with the interpretation that gaze discrimination is facilitated when joint attention is established. This finding therefore extends previous work showing that engaging in joint attention facilitates a range of social cognitive processes.
\end{abstract}




\section{Joint attention facilitates observed gaze direction discrimination}

Where other people look can inform us about our environment and the person whose gaze we observe (see Frischen, Bayliss \& Tipper, 2007 for review). As the eyes are such rich signals, humans are highly skilled at discerning the gaze direction of others and have specialised neural systems for visual and social processing of gaze (Perrett, Hietanen, Oram \& Benson, 1992; Nummenmaa \& Calder, 2009). We are particularly sensitive to being looked at: faces with direct gaze capture our attention, lead to increased arousal, and are recognised more efficiently (e.g. Senju \& Hasegawa, 2005; see Hamilton, 2016 for review). However, averted gaze is also socially informative - we can follow the gaze of others to establish joint attention and therefore learn about the gazer and the environment more generally (Moore \& Dunham, 1995; see Frischen et al., 2007 for review). The present work investigates how we code averted gaze cues that are presented non-foveally, a situation that real-world joint attention scenarios would necessitate. Prior work has illustrated that social cognition systems are engaged when viewing non-foveal gaze cues (e.g. Böckler, van der Wel, \& Welsh, 2014; Friesen \& Kingstone, 2003). Yet it remains unclear the ways in (and extent to) which these gaze signals may be compromised.

In a notable recent line of work, participants have been asked to discriminate the gaze direction of peripherally presented faces (Cañadas \& Lupiáñez, 2012; Jones, 2015; Marotta, Román-Caballero, \& Lupiáñez, 2018). In these experiments, participants fixated a central fixation cross and a face was presented to the left or right of fixation with averted gaze looking leftwards or rightwards. Participants were faster at left/right gaze discrimination for gaze oriented 'inwards', towards the centre of the scene (and the location of the participants' fixation). This facilitation for inwards-orientated observed gaze has been interpreted as resulting from the 'inward' gaze being misattributed by participants as direct gaze (Cañadas \& Lupiáñez, 2012). This interpretation is consistent with previous literature on gaze 
perception. Specifically, it has been clearly demonstrated that the human gaze perception system is biased towards interpreting gaze as being direct; under conditions of perceptual uncertainty, we will assume we are being looked at (Mareschal, Calder \& Clifford, 2013). A bias for interpreting ambiguous gaze as direct is advantageous because missing direct gaze signals can be very costly. This is because direct gaze could precede an approach behaviour and the initiation of a positive or negative social interaction. Thus, the current interpretation of facilitated discrimination of peripheral gaze cues that look 'inwards' as being related to the misattribution of direct gaze has significant merit.

An alternative interpretation of the facilitation of gaze discrimination for inward gazing faces can be proposed. In the context of the studies on this effect thus far, a peripherally-located face that looks inwards with regards to the scene is also looking towards the location that participants are fixating. Thus, if we assume that the participant does not misattribute the observed inwards gaze as directed towards themselves but instead interprets it as directed towards the fixation cross to which they are also looking, then the participant and the onscreen face are engaged in an episode of simulated joint attention. Conversely, when the eyes look away from where the participant is looking, gaze discrimination does not benefit because joint attention is not established. There is good evidence that engaging in a simulated joint attention episode facilitates processing of the elements of the joint attention scenario. For example, in the context of exploring 'gaze leading', where participants actively shift their fixation position during a trial, we have shown that peripheral faces displaying averted gaze towards a participants fixation location capture attention (Edwards, Stephenson, Dalmaso \& Bayliss, 2015; see also Bayliss, Murphy, Naughtin, Kritikos, Schilbach, \& Becker, 2013; Stephenson, Edwards, Howard \& Bayliss, 2018). These studies on 'gaze leading' employed tasks involving eye movements by the participants, but it could be that facilitated processing of faces engaging in joint attention could emerge even in passively- 
established simulated joint attention scenarios. If so, a 'joint attention' account of the advantage for the discrimination of inward-oriented gazing faces could explain the findings of Cañadas and Lupiáñez (2012).

The present work aimed to discriminate between these two alternative accounts of the gaze discrimination facilitation effect for inward looking faces. We describe two experiments where participants made speeded left/right gaze direction discriminations of peripheral faces that looked leftwards or rightwards. As well as replicating previous work by having participants fixate a central location, we included other trials where the participants fixation location was positioned eccentrically in the scene. If gaze discrimination is facilitated by the misattribution of inward gaze as direct gaze, then the manipulation of the participants fixation target will not modulate the bias for inward-looking gaze because these faces will still have the same perceptual qualities that might induce an impression that they are looking at the participant. Alternatively, if peripheral gaze discrimination is facilitated by joint attention towards a common shared referent, then facilitation of gaze direction discrimination should be modulated by fixation position and should be present only where the face is looking at the same location as the participant, whether this is a central or non-central location.

\section{Experiment 1}

Experiment 1 closely replicates the procedures of Cañadas and Lupiáñez (2012), with one main modification. Participants were sat centrally with regards to the display and made speeded gaze direction discriminations of faces appearing to the left or right of centre. We included trials where the fixation cross was positioned centrally such that inward gaze was directed towards the participants fixation location (see Figure 1, panels A-B). These conditions directly replicate the procedure of Cañadas and Lupiáñez, thus we predict facilitated gaze discrimination for inward (Figure 1, panel A) over outward (Figure 1, panel B) gaze. We also introduced new trial types where the fixation cross was positioned to the 
periphery of the display (Figure 1, panels C-F). Having the fixation cross appearing at the far left or right of the display meant that we could pit the two competing hypotheses regarding the gaze discrimination facilitation against each other: If peripheral gaze identification is facilitated because inward facing gaze is misattributed as direct gaze, inward-oriented gaze should be identified more rapidly even when participants are looking elsewhere (e.g. Figure 1, panel D). Conversely, if gaze discrimination is facilitated due to joint gaze, we predicted identification of peripheral gaze would be facilitated for outward oriented gaze that is towards the participant's fixation location (e.g. Figure 1, panel C). Closely replicating the procedures of Cañadas and Lupiáñez, where faces appeared to the left or right of centre with regards to the display and manipulating only the fixation location of the participant meant that we also had trials where the to-be-judged face was on the opposite side of the display to the fixation location (Figure 1, panels E-F). While these trials balance the design, they do not help distinguish between the two competing hypotheses of interest, as in these trials, inward gaze and joint attention always co-occurred. We therefore analyse them separately from the trials with faces in more immediate proximity to the fixation target. However, data from these trials could help us answer a separate and interesting question regarding the limits of visual eccentricity relating to the facilitation of gaze discrimination for inward gaze. We anticipated that the facilitation of discriminating inward gaze would rely on joint gaze, and thus be reversed when participants fixated a non-central fixation cross, thereby illustrating a critical role of joint gaze in facilitating gaze processing. 

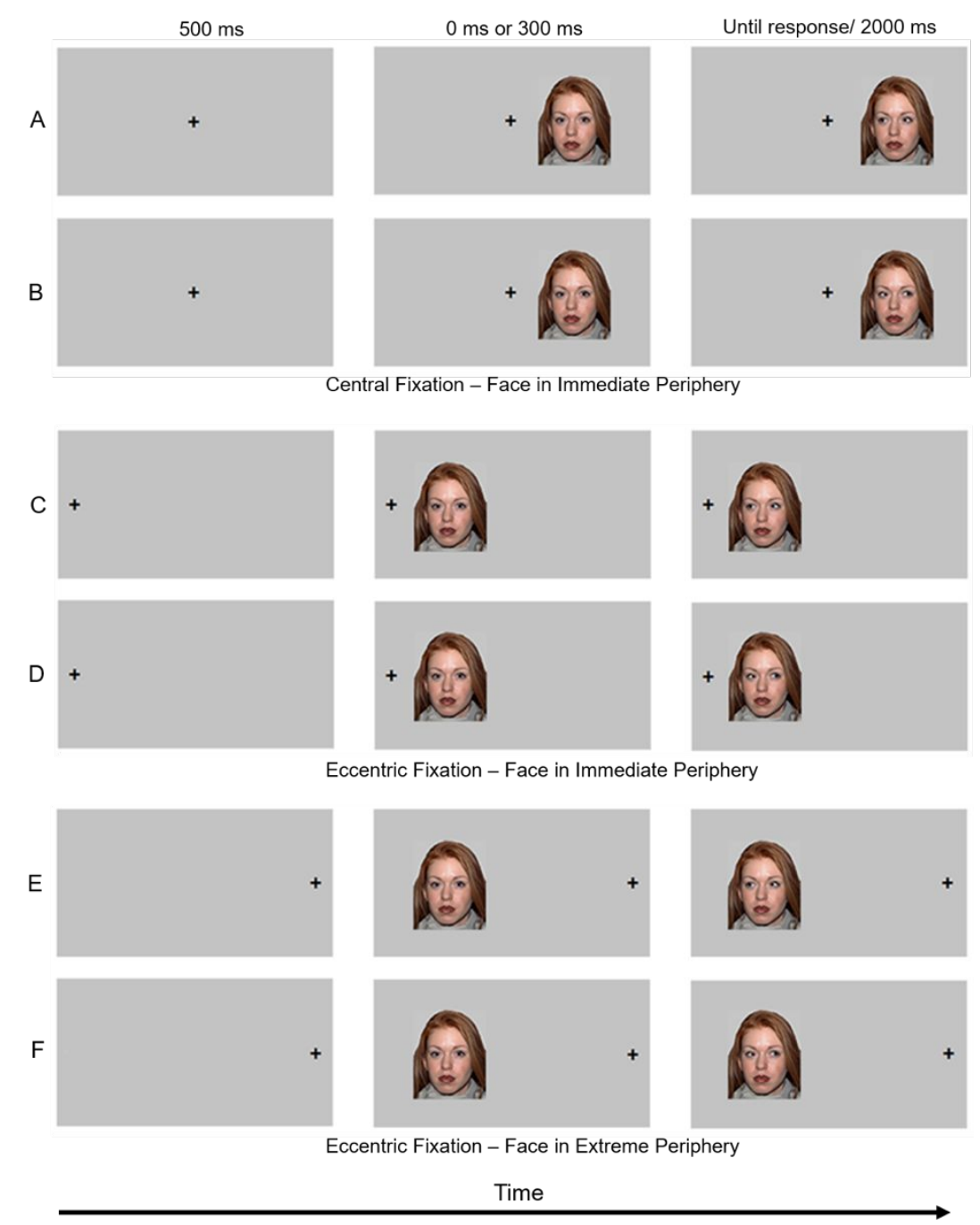

Figure 1. Example procedure in Experiment 1. Trials started with a fixation cross shown for $500 \mathrm{~ms}$, which participants fixated, presented centrally (A, B) or peripherally to the left or right (C-F). Next a face was presented to the left or right of centre with straight gaze for $0 \mathrm{~ms}$ (i.e. not presented) or for $300 \mathrm{~ms}$ as per SOAs selected by Cañadas \& Lupiáñez (2012), after which the face displayed averted gaze towards $(\mathrm{A}, \mathrm{D}, \mathrm{F})$ or away from $(\mathrm{B}, \mathrm{C}, \mathrm{E})$ the centre of the screen. Participants made speeded discrimination of gaze direction, pressing the ' $Z$ ' key to indicate leftward gaze or the ' $M$ ' key to indicate rightward gaze. Note that on trials with SOA $0 \mathrm{~ms}$ no straight gaze was presented. Stimuli are not to scale. 


\section{Method}

In both experiments we have reported how we determined our sample size, all data exclusions (if any), all manipulations, and all measures we have collected (see Simmons, Nelson \& Simonsohn, 2012).

Participants. We targeted a sample of $\mathrm{N}=20$ to closely match the original demonstration of the effect of interest. Informed consent was obtained from 20 undergraduate students ( 2 men), from the University of East Anglia (age; $M=21.5$ years, $S D=6.0$ years) to participate in the experiment in exchange for course credit or payment. All were naïve as to the purpose of the experiment.

Materials. Stimuli consisted of 24 colour photographs of 8 identities (4 male, 4 female; 3 images per identity) with a neutral expression, taken from the NimStim photo set (Tottenham et al., 2009). The original image $(8 \mathrm{~cm} \mathrm{x} 10.5 \mathrm{~cm})$ of each identity had straight gaze, and had been edited to create 2 new images containing gaze to the far left and far right, respectively. E-Prime 2.0 software was used to create the experimental program, which allowed for automated stimuli presentation \& data collection. Each participant was run on a standard Dell desktop PC with a 17" monitor running at 640×480-pixel resolution.

Design. Our design kept the perceptual stimuli available to participants as closely aligned to prior work as possible (e.g. Cañadas \& Lupiáñez, 2012). However, the design choice to manipulate the position of the participants fixation target to appear not only in the centre but also at the periphery of the display meant that some trials would result in the to-bejudged face appearing on the opposite side of the display to that of the participants fixation target (see Figure 1, panels E-F). Importantly, these trials do not help us to answer our main research question regarding the distinction of perceiving direct gaze vs prioritising joint gaze partners, because gaze towards the participants fixation target would always also be 'inward'. However, these trials do allow us to assess a separate and interesting question regarding 
whether the current effects extend to faces that are processed in the extreme periphery. Thus, we analysed data from these trials separately to the main analysis of the other trial types (see Figure 1, panels A-D). Thus, a 2 (Fixation Position: Central, Non-central) $\times 2$ (SOA: 0ms, $300 \mathrm{~ms}) \times 2$ (Gaze: Inward, Outward) within subjects design was used in order to assess the critical research question, including only trials where the face appeared at equivalent distances to the fixation location. Trials where the face was on the opposite side of the display to the fixation location were analysed separately using a separate 2 (SOA: 0ms, $300 \mathrm{~ms}) \times 2$ (Gaze: Inward, Outward) within subjects analysis. Mean reaction time (RT) and accuracy $(\%)$ were measured.

Procedure. Participants sat approximately $60 \mathrm{~cm}$ from the screen and were required to make speeded discrimination of the direction (left or right) of onscreen averted gaze, pressing the ' $\mathrm{Z}$ ' key or ' $\mathrm{M}$ ' key with left and right index fingers, respectively. Participants started each trial fixating the fixation cross (blocked by position; left (16.5 cm from centre), centred, right (16.5 cm from centre) on a silver background for $500 \mathrm{~ms}$ (see Figure 1). Block sequence was randomised. Next, a picture of a face with straight gaze was presented either to the left or to the right of the screen $(7.5 \mathrm{~cm}$ from Centre) for 0 or $300 \mathrm{~ms}$. Finally, the same picture with the eyes gazing either to the right or to the left was presented at the same location until response or $2000 \mathrm{~ms}$ had elapsed. Note: in cases of the $0 \mathrm{~ms} \mathrm{SOA}$, the face appeared with averted gaze only, whereas with SOA of $300 \mathrm{~ms}$ the faces 'looked' towards the left or right. A practice block consisting of 48 randomly selected trials preceded three experimental blocks of 128 trials each (one per fixation point position).

\section{Results}

We conducted the same analyses on both mean accuracy data and mean reaction time data. Responses above or below $3 S D$ of participants means were removed ( $1.5 \%$ data in each experiment) prior to calculation of reaction time means for each condition. Below we report a 
2 (Fixation Position; Central, Non-central) $\times 2($ SOA; 0, 300) $\times 2$ (Gaze: Inward, Outward $)$ ANOVA to assess whether peripheral gaze discrimination is facilitated for inward gaze or joint attention. We then report a separate $2(\mathrm{SOA} ; 0,300) \times 2$ (Gaze: Inward, Outward) ANOVA to assess whether peripheral gaze identification facilitation extends to greater visual eccentricities. The analysis was identical in Experiment 2. Mean accuracy and mean reaction times for all trial types can be found in Table 1. 
Table 1. Means and Standard Deviations (in parentheses) for reaction times (ms) and accuracy (\%) in all conditions, in Experiments 1 \& 2.

Inward denotes trials where the onscreen face presented gaze directed towards the centre of the display, Outward denotes trials where displayed onscreen gaze was directed away from centre.

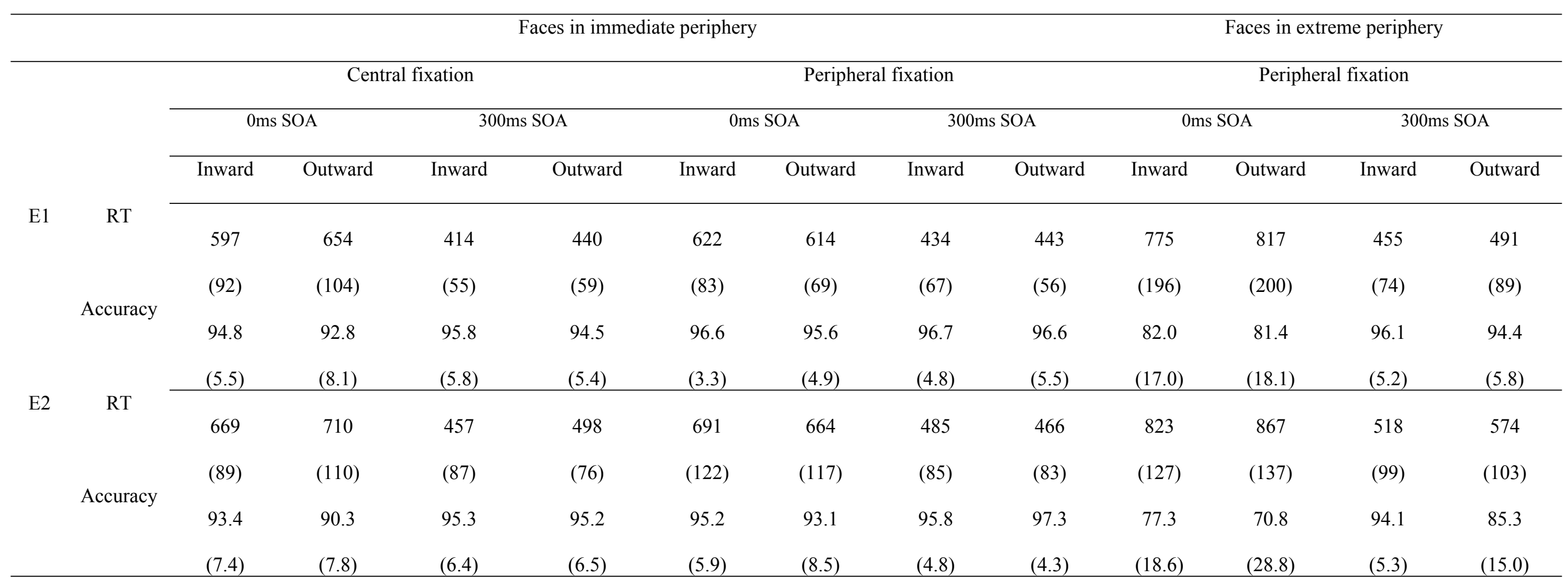




\section{Accuracy.}

Faces in immediate periphery. A 2 (Fixation Position; Central, Non-central) $\times 2$ $(\mathrm{SOA} ; 0,300) \times 2$ (Gaze: Inward, Outward) ANOVA revealed no significant effect of SOA, $F(1,19)=1.21, p=.286, \eta_{p}^{2}=.06$, or Gaze, $F(1,19)=2.05, p=.168, \eta_{p}^{2}=.098$. However there was a significant effect of Fixation Position, $F(1,19)=4.94, p=.039, \eta^{2}{ }_{p}=.21$, as accuracy was higher when the Fixation Position was Non-central (96.4\%) than Central (94.5\%). No interactions reached significance $\left(F{ }^{\prime}<<.637, p\right.$ 's $\left.>.435\right)$.

Faces in extreme periphery. A second 2 (SOA; 0, 300) $\times 2$ (Gaze: Inward, Outward) ANOVA was conducted on responses from trials where the face appeared on the opposite side of the screen to the object. The only significant effect was of SOA, $F(1,19)=16.11$, $p=.001, \eta_{p}^{2}=.46$, indicating that the accuracy was better at the longer $(95.2 \%)$ than at the shorter $(81.7 \%)$ SOA. Neither the Gaze, $F(1,19)=.60, p=.447, \eta_{p}^{2}=.031$, nor the interaction of Gaze $\times$ SOA, $F(1,19)=.16, p=.693, \eta_{p}^{2}=.008$, were significant.

\section{Reaction times.}

Faces in immediate periphery. A 2 (Fixation Position; Central, Non-central) $\times 2$ $(\mathrm{SOA} ; 0,300) \times 2($ Gaze: Inward, Outward) ANOVA on reaction times $(\mathrm{RTs})$ revealed a significant effect of Gaze, $F(1,19)=10.60, p=.004, \eta^{2}=.36$, indicating that RTs were faster when the face was looking Inwards $(517 \mathrm{~ms})$, than Outward (538ms). There was also a significant effect of SOA, $F(1,19)=223.77, p<.001, \eta^{2}{ }_{p}=.92$, indicating that RTs were faster at the longer $(433 \mathrm{~ms})$ than shorter $(622 \mathrm{~ms})$ SOA. There was no main effect of Fixation Position, $F(1,19)=.031, p=.861, \eta^{2}{ }_{p}=.002$, SOA $\times$ Gaze interaction, $F(1,19)=1.03, p=.323$, $\eta^{2}{ }_{p}=.051$, nor Fixation Position $\times$ SOA interaction, $F(1,19)=3.367, p=.082, \eta^{2}=.15$. However, the critical Gaze $\times$ Fixation Position interaction was significant, $F(1,19)=12.018$, $p=.003, \eta_{p}^{2}=.387$, indicating that the facilitation for Inward Gaze is sensitive to where the participant is looking (Fixation Position). Indeed, whereas responses were faster for Inward 
Gaze when participants were fixating a central location (when joint gaze occurs; $41 \mathrm{~ms}$ advantage, $t(19)=-4.301, p<.001, d z=.96)$, no such facilitation emerged for trials where the participants were fixating a non-central location (non-joint attention; 0ms advantage, $t(19)=.035, p=.972, d z=.008)$. The 3-way interaction was also significant, $F(1,19)=11.219$, $p=.003, \eta_{p}^{2}=.371$; when fixating centrally, participants discriminated Inward Gaze more rapidly than Outward Gaze at both SOAs. However, when fixating a non-central location, Inward Gaze was identified more rapidly than Outward Gaze for trials with the longer SOA, but the reverse pattern emerged for the shorted SOA.

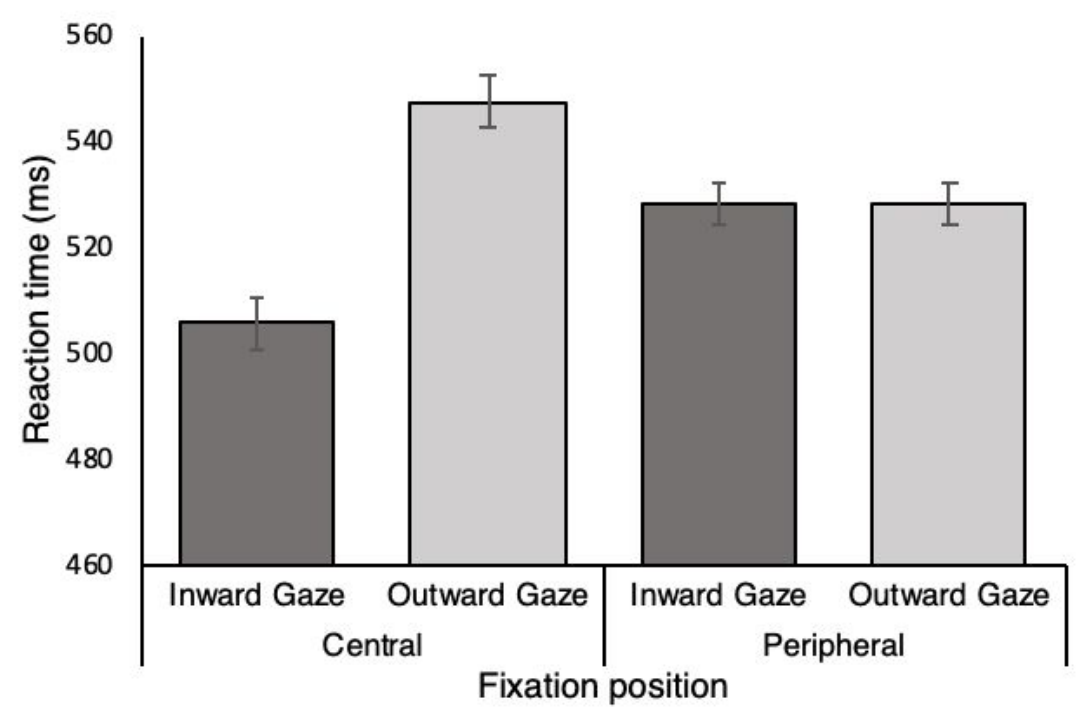

Figure 2. Mean reaction times (RT) for correctly discriminating displayed gaze direction in Experiment 1, divided by Fixation Position and Gaze direction. Error bars represent withinsubject standard error of the mean (Loftus \& Masson, 1994).

Faces in extreme periphery. A separate $2(\mathrm{SOA} ; 0,300) \times 2$ (Gaze: Inward, Outward) ANOVA was conducted on responses from trials where the face appeared on the opposite side of the screen to the fixation cross. There was a significant effect of SOA, $F(1,19)=116.23, p<.001, \eta_{p}^{2}=.86$, due to faster responses at the longer (473ms), than shorter (796ms), SOA. The main effect of Gaze was also significant, $F(1,19)=12.091, p=.003, \eta_{p}^{2}=$ 
.39 , as RTs were faster on Inward $(615 \mathrm{~ms})$, than Outward $(654 \mathrm{~ms})$, trials. The SOA $\times$ Gaze interaction was not significant, $F(1,19)=.15, p=.669, \eta_{p}^{2}=.01$.

\section{Discussion}

Left/right discrimination of gaze direction was facilitated for inward gaze towards a centrally presented fixation cross, directly replicating previous work (Cañadas \& Lupiáñez, 2012; Jones, 2015; Marotta et al., 2018). However, this effect was unreliable when the participants were not attending to a centrally presented fixation cross, but instead fixating a more peripheral location, illustrating the importance of the joint gaze context for the currently investigated gaze discrimination modulation. Our secondary analysis of gaze discrimination responses of faces in the extreme periphery showed that gaze that is directed inwards and towards the fixation location of the participant is discriminated more fluently than gaze 'looking' elsewhere. Thus, we also extend previous work by also showing that the current effects are robust to a range of visual eccentricities.

It is interesting that there was no gaze direction discrimination for either inward or outward gaze when participants fixated a non-central cross. This could be interpreted as being due to the effect of joint gaze being cancelled out by an opposite faciliatory effect. For example, scene perception literature suggests a special roll for inward facing gaze (see Chen, Colombatto \& Scholl, 2018). Alternatively, the misattribution of averted gaze as eye-contact may play some role, which the joint gaze effect is unable to overpower in this instance. Intriguingly, while collapsing the data by SOA appears to show a 'disrupted' effect for noncentral fixation location trials, the 3-way interaction shows that actually the data replicated the 'inward' bias at the longer SOA, but completely reversed at the shorter SOA. This pattern of data suggests that joint attention, as opposed to 'inward gaze', is rapidly identified and responded to (cf. Edwards et al., 2015). Nevertheless, at the longer SOA, where the face initially displays straight gaze before 'looking' to the left or right, gaze discriminations are 
facilitated for inward gaze. This may suggest that inward gaze is more likely to be misinterpreted as direct gaze if it proceeds straight gaze. This explanation is of course speculative and this flipping of effects by SOA in the present experiment is difficult to reconcile with previous work or our predictions regarding the importance of joint attention. In Experiment 2 we increase the salience of the joint attention context in order to further examine the role joint attention processes may have for peripheral gaze discriminations.

\section{Experiment 2}

The current work is interested in investigating the extent to which a previously reported gaze discrimination modulation may relate to joint attention processes. Our prediction of this account is that peripheral gaze direction discrimination will be enhanced for joint gaze (verses non-joint gaze), which may rule out prior explanations relating to misattributing inward gaze as being direct gaze. The data of Experiment 1 are not totally clear, as although the discrimination benefit for inward gaze was modulated by the fixation location of a participant - suggesting a crucial role for joint attention - we found data aligning with each of the two competing hypotheses across the two SOA conditions when participants fixated a peripheral location.

Joint attention is necessarily object-based; the inclusion of an object distinguishes joint attention from mere gaze following (Emery, 2000). Indeed, many studies investigating gaze cueing and gaze leading have shown enhanced effects when using images of real-world objects as to-be gazed-at stimuli over and above simple geometric stimuli such as fixation crosses (e.g. Bayliss \& Tipper, 2005; Edwards et al., 2015). Therefore, in Experiment 2, we replaced the fixation cross with images of real-world objects to assess the extent to which the current effects relate to incidental joint gaze processing (see Figure 3). We predicted that this methodological modification would enhance the consequences of joint gaze in this paradigm 
and show a clearer advantage for processing gaze that establishes joint attention than was revealed in the equivocal data of Experiment 1.

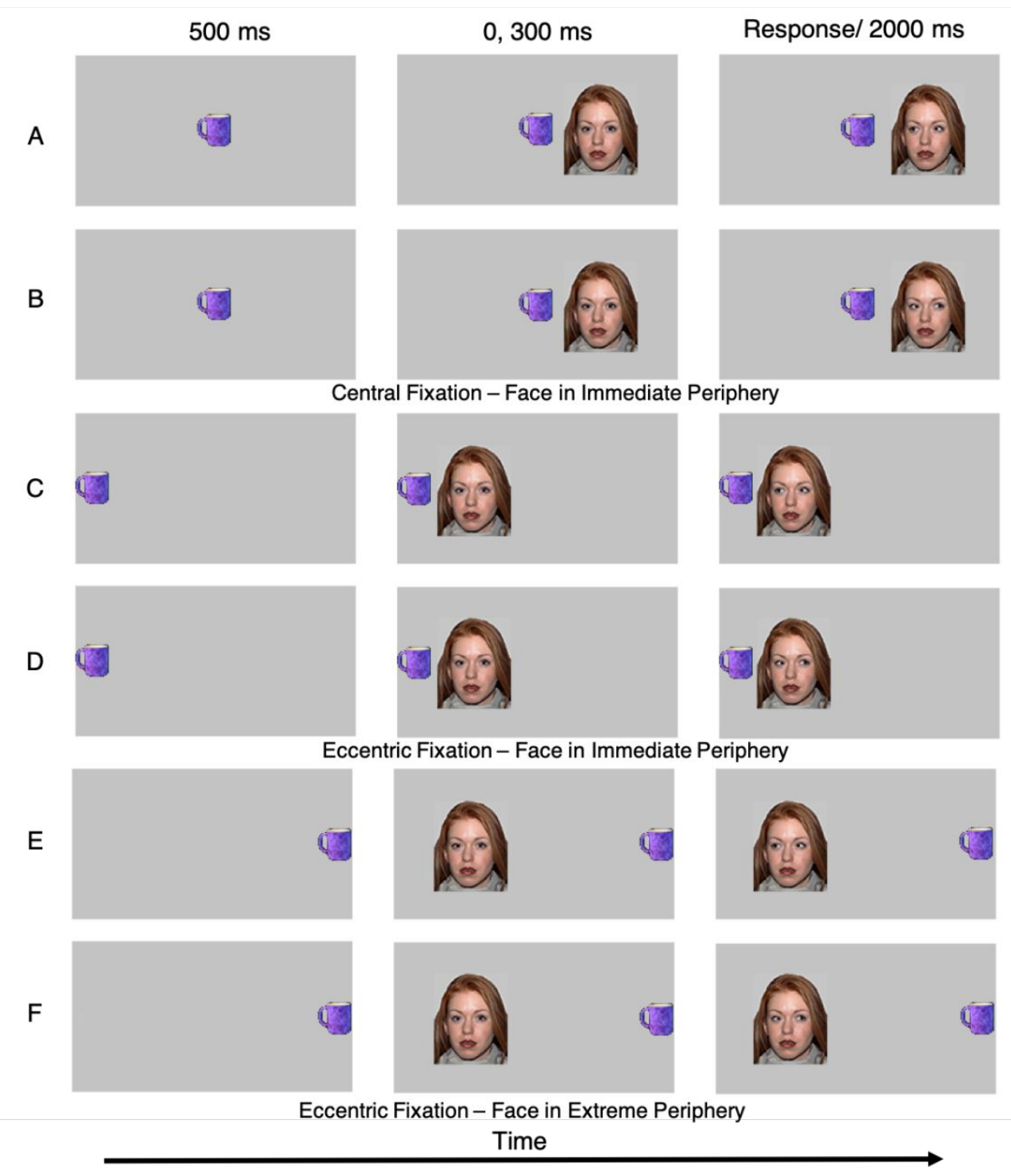

Figure 3. Example procedure in Experiment 2. Trials started with an object image (randomly selected per trial from 16 options) shown for $500 \mathrm{~ms}$, which participants fixated, presented centrally $(A, B)$ or peripherally to the left or right (C-F). Next a face was presented to the left or right of centre with straight gaze for $0 \mathrm{~ms}$ (i.e. not presented) or for $300 \mathrm{~ms}$ as per SOAs selected by Cañadas \& Lupiáñez (2012), after which the face displayed averted gaze 
towards $(\mathrm{A}, \mathrm{D}, \mathrm{F})$ or away from $(\mathrm{B}, \mathrm{C}, \mathrm{E})$ the centre of the screen. Participants made speeded discrimination of gaze direction, pressing the ' $Z$ ' key to indicate leftward gaze or the ' $\mathrm{M}$ ' key to indicate rightward gaze. Note that on trials with SOA 0ms no straight gaze was presented. Stimuli are not to scale.

\section{Methods}

Participants. Informed consent was obtained from 21 students (4 males), from the University of East Anglia (Age; $M=19.4$ years, $S D=0.9$ years) to participate in the experiment in exchange for course credit or payment. All were naïve as to the purpose of the experiment.

Materials. The set-up was identical to Experiment 1, except that the fixation crosses were replaced by object images (see Figure 3). Object images were taken from the set used by Bayliss, Bartlett, Naughtin \& Kritikos (2011) and consisted of 16 different objects usually found in the kitchen.

Design \& Procedure. Identical to Experiment 1, except that an object image (randomly selected per trail from 16 options) was in place of the fixation cross.

\section{Results}

Analyses were conducted as in Experiment 1. 1.5\% of data were removed as outliers. Data from one participant was excluded because she or he did not follow instructions (chance performance) hence $\mathrm{N}=20$ hereafter. Accuracy and reaction times can be found in Table 1 .

\section{Accuracy.}

Faces in immediate periphery. A 2 (Object Position; Central, Non-central) $\times 2$ (SOA; $0,300) \times 2$ (Gaze: Inward, Outward) ANOVA revealed a significant effect of SOA, $F(1$, $19)=7.81, p=.012, \eta_{p}^{2}=.29$, indicating that accuracy was better at the longer $(95.9 \%)$ than at the shorter (93.0\%) SOA. There was also a reliable effect of Object Position, $F(1,19)=5.32$, $p=.033, \eta_{p}^{2}=.22$, as accuracy was higher for Non-central (95.4\%) than Central (93.6\%) object fixations. There was no effect of Gaze, $F(1,19)=.31, p=.585, \eta_{p}^{2}=.016$. None of the two-way 
interactions reached significance $\left(F{ }^{\prime}<<2.4, p\right.$ 's $\left.>14\right)$. The 3-way interaction was significant, $F(1,19)=5.18, p=.035, \eta_{p}^{2}=.22$, due to higher accuracy for Inward over Outward gaze at both fixation locations for the shorter SOA, but for the longer SOA accuracy did not differ between gaze direction for Central Fixation but was higher for Outward gaze at the Noncentral fixation.

Faces in extreme periphery. A second 2 (SOA; 0, 300) $\times 2$ (Gaze: Inward, Outward) ANOVA was conducted on responses from trials where the face appeared on the opposite side of the screen to the object. The only significant effect was of SOA, $F(1,19)=27.78$, $p<.001, \eta_{p}^{2}=.59$, indicating that the accuracy was better at the longer $(89.7 \%)$ than at the shorter (74.1\%) SOA. The main effect of Gaze did not reach statistical significance, $F(1,19)=3.85, p=.065, \eta_{p}^{2}=.17$, but the data trend showed higher accuracy for Inward $(83.6 \%)$ than Outward (76.6) Gaze. The Gaze $\times$ SOA interaction, $F(1,19)=.22, p=.643, \eta_{p}^{2}=$ .012 , was not significant.

\section{Reaction times.}

Faces in immediate periphery. A 2 (Object Position; Central, Non-central) $\times 2(\mathrm{SOA}$; $0,300) \times 2$ (Gaze: Inward, Outward) ANOVA on reaction times (RTs) revealed a significant effect of SOA, $F(1,19)=329.16, p<.001, \eta^{2}=.95$, indicating that RTs were faster at the longer $(477 \mathrm{~ms})$ than shorter $(683 \mathrm{~ms})$ SOA. There was no main effect of Object Position, $F(1,19)=.22, p=.648, \eta_{p}^{2}=.011$, nor Gaze, $F(1,19)=1.85, p=.189, \eta^{2}{ }_{p}=.09$. Critically, there was a significant Object Position $\times$ Gaze interaction $F(1,19)=11.73, p=.003, \eta^{2}{ }_{p}=.38$, indicating that the facilitated discrimination for Inward Gaze was sensitive to where the participant was looking (Object Position). Indeed, whereas responses were faster for Inward Gaze when that gaze was towards the participants own fixation location (when joint attention occurs; $43 \mathrm{~ms}$ advantage, $t(19)=-3.13, p=.006, d z=.74)$, the reverse pattern emerged for trials where the participant was fixating a Non-central location $(23 \mathrm{~ms}$ disadvantage, $t(19)=2.46$, 
$p=.024, d z=-.29)$, which indicates a reversal of the 'inward' effect and supports the interpretation that joint gaze drives the gaze discrimination modulations reported. There were no other significant interactions: Object Position $\times$ SOA interaction $F(1,19)=.753, p=.396$, $\eta_{p}^{2}=.038 ;$ Gaze $\times$ SOA interaction $F(1,19)=.087, p=.771, \eta_{p}^{2}=.005,3$-way interaction, $F(1,19)=0.384, p=.543, \eta_{p}^{2}=.020$.

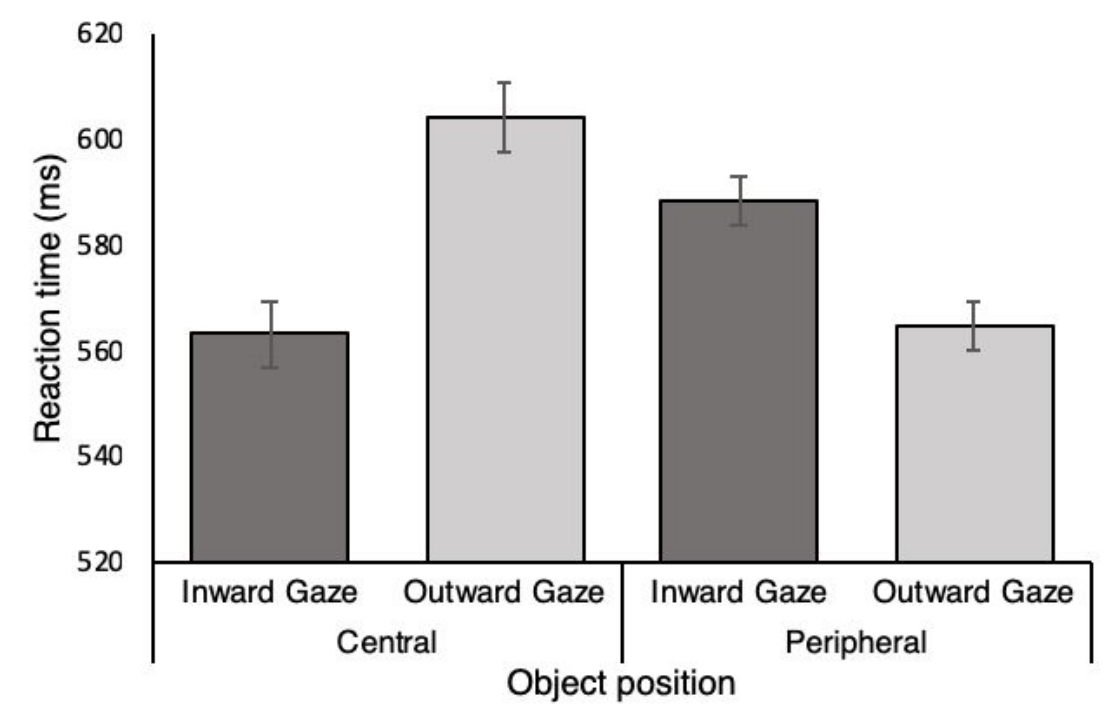

Figure 4. Mean reaction times (RT) for correctly discriminating displayed gaze direction in Experiment 2, divided by Object position and type of Gaze. Error bars represent within-subject standard error of the mean (Loftus \& Masson, 1994).

Faces in extreme periphery. A second, separate, $2(\mathrm{SOA} ; 0,300) \times 2($ Gaze: Inward, Outward) ANOVA was conducted on responses from trials where the face appeared on the opposite side of the screen to the object. There was a significant effect of SOA, $F(1,19)=182.59, p<.001, \eta_{p}^{2}=.91$, owing to faster responses at the longer $(546 \mathrm{~ms})$, than shorter $(845 \mathrm{~ms})$, SOA. The main effect of Gaze was also significant, $F(1,19)=17.37, p=.001$, $\eta_{p}^{2}=.48$, as RTs were faster on Inward $(671 \mathrm{~ms})$, than Outward $(721 \mathrm{~ms})$, trials. The SOA $\times$ Gaze interaction was not significant, $F(1,19)=.63, p=.436, \eta^{2}{ }_{p}=.03$. 


\section{Discussion}

As in Experiment 1, participants were faster to discriminate leftward and rightward gaze presented in the periphery when that gaze was directed inward towards the central fixation object, over multiple visual eccentricities, thereby also replicating and extending prior investigations (Cañadas \& Lupiáñez, 2012; Jones, 2015; Marotta et al., 2018). However, this pattern of response was reversed when participants fixated a more peripheral object, with outward rather than inward gaze being discriminated more rapidly. Unlike Experiment 1 this data is much clearer, with no interaction with SOA. Taken together, the present data therefore supports the interpretation that peripheral gaze discrimination is facilitated by joint attention towards images of real-world objects, exemplifying the importance of object-related processing within simulated joint attention scenarios.

\section{General discussion}

In both experiments, we replicated the previously reported finding that inward gaze towards a participants current fixation target is discriminated more rapidly than gaze away from that target (Cañadas \& Lupiáñez, 2012; Jones, 2015; Marotta et al., 2018). However, when a participant fixated a non-central location the facilitation for discriminating inward gaze becomes less reliable (Experiment 1). Importantly, we show that the opposite pattern of responses can emerge when participants fixate a non-central object image, such that outward gaze is discriminated more rapidly when that gaze is directed towards the participant's fixation location (Experiment 2). This latter finding is incompatible with the idea that peripheral gaze discrimination is facilitated by inward gaze because it is misattributed as direct gaze. Instead, this data suggests that joint overt visual attention leads to facilitated gaze discrimination.

It is important that we clarify that in the current paper we define joint attention as instances of joint overt visual attention (or joint gaze) and do not necessarily imply any 
higher-level (e.g. mentalising) mechanisms being involved. The definition of joint attention can vary. The end point of the encounter resulting in joint gaze is sufficient for some (e.g. Emery, 200; Edwards et al., 2015), while others include an initial direct gaze (simulated mutual gaze) phase as part of the bout of joint attention (e.g. Schilbach, Eickhoff, Cieslik, Shah, Fink \& Vogeley, 2010; Redcay, Kleiner \& Saxe, 2012). Indeed, while some real-world joint gaze encounters might first involve the two parties looking at one another as an ostensive cue to the upcoming joint gaze encounter, joint attention could also occur incidentally, for example when a conspecific 'happens' to look at what we are looking at when we did not intend for them to do so. In the latter case, it would still be beneficial to notice and track who has followed our gaze (Edwards et al., 2015; Dalmaso et al., 2016). In the current work some trials included initial direct gaze, while other did not. In Experiment 2, where our effects were most clear, whether or not initial direct gaze occurred had no impact on a participant's ability to identify gaze direction. This speaks to the effects observed as likely rapid and low-level responses to the peripheral gaze cue which is directed to the participants own fixation target (Edwards et al., 2015). Nevertheless, future work may benefit from investigating the extent to which the attentional prioritisation (Edwards et al., 2015) and response facilitation shown in the present work for peripherally presented joint gaze might relate to a mental perspective taking mechanism involving computing whether another agent is sharing our perspective.

The prioritisation of peripheral faces that look to a participant's fixation target has been shown elsewhere (Edwards et al., 2015). Notably, attention prioritisation of peripheral faces that gazed towards a participant's fixation target was sensitive to the target context: joint gaze faces were preferentially attended when participants fixated object images, but not when the fixation target was a mere fixation cross. This pattern of data fits with the current work, where the null effect of gaze direction in Experiment 1 for non-central fixation targets 
could be interpreted as an insensitivity to the joint gaze context involving fixation crosses.

However, the data of the current Experiment 1 was more nuanced than merely 'null': the data flipped between the shorter and longer SOA, which makes interpretation from this intriguing data less straightforward.

We cannot rule out the potential for multiple parallel processes in the current data. For example, it is entirely possible that inward gaze and gaze that results in joint attention are both facilitative and happen to cancel one another out in the non-central fixation condition of Experiment 1. Indeed, research focussing on aesthetics has shown that agents that are facing inwards within a scene are preferred to agents facing outwards; the inward bias (see Chen, Colombatto \& Scholl, 2018) and ambiguous figures are preferentially perceived as the variant that is 'looking' inward (Chen \& Scholl, 2014). It is possible that this affective preference is associated with facilitated processing (i.e. perceptual fluency, see Reber, Winkielman \& Schwar, 1998).

It seems the emerging evidence, as well as the present work, suggests that the misattribution of direct gaze from observed inward gaze is unlikely to drive the current effects. For example, there is conflicting evidence regarding how approach and avoidance emotions in the gazing face affects the processing of off-centre inward gaze (cf. Jones, 2015; Torres-Marin, Carretero-Dios, Acosta \& Lupiáñez, 2017). Further, Torres-Marin et al., found that participants level of Gelotophobia (fear of being laughed at), which should relate to the sensitivity to direct gaze, did not impact gaze identification in a paradigm similar to that used here. Taken together with our demonstration of the importance of the looked-at object, it therefore appears that the misattribution of direct gaze can no longer account for these interesting data.

We interpret the current effects as relating to the gaze leading effect as the attentional prioritisation of joint gaze partners could account for the gaze discrimination advantage we 
report here (see Edwards et al., 2015). It is notable that the gaze leading effect appeared to be social in nature as for example the magnitude of the effect was sensitive to individual differences in self-reported autism-like traits, only emerged in object-based encounters, and did not replicate with arrow cues (Edwards et al., 2015). Strikingly, whereas there is an abundance of work showing comparable attention orienting responses to gaze and arrow cues (see Frischen et al., 2007 for review), Marotta et al (2018) also showed that peripheral gaze discrimination for inward cues did not replicate with arrow stimuli - the reverse pattern emerged, which is in line with a 'spatial congruency' account. Thus, we can now point to two lines of work with different paradigms - the present (the work on which we build e.g. Marotta et al., 2018) and that on gaze leading (e.g. Edwards et al., 2015) - that illustrate cases where gaze and arrows produce opposite faciliatory effects. This is in stark contrast to the extant literature and opens up an exciting avenue for future work to elucidate the boundary conditions where arrows and gaze are and are not similarly faciliatory. For example, it may only be that when the potentially more socially complex process of working out whether someone is looking at the same thing we are looking at - as opposed to merely ascertaining where they are looking - is engaged that gaze becomes distinguishable from arrows in terms of their impacts of attention-related processes.

As mentioned above, prior work using the same basic paradigm as the current investigation, but with only a central fixation location has shown arrows to produce opposite facilitation to gaze - e.g. left arrows are identified more rapidly when they point left - in line with a spatial congruency account (Marotta et al., 2018; see Cañadas \& Lupiáñez, 2012). We thank a reviewer for therefore pointing out that our current paradigm whereby the fixation location is manipulated could allow future investigations to assess how the change in the fixation location within a scene affects the standard spatial congruency effect - a question that to our knowledge is untested. Indeed, it is not clear how the faces or arrows (and fixation 
targets) within the current work are coded - e.g. whether spatially coded in reference to the overall screen, the fixation point or both (Danziger, Kingstone, \& Ward, 2001 see also Behrmann \& Tipper, 1999; Farah, Brunn, Wong, Wallace, \& Carpenter, 1990).

To conclude, we have shown that peripheral gaze discrimination is facilitated when joint attention occurs. This effect appears robust to multiple visual eccentricities, but is sensitive to the social context of the object-centred interaction. These findings may offer a new light with which to view previous works that have used non-central faces as gaze cues. The social specificity of this effect and its link to the gaze leading effect remain interesting avenues for future investigations. 
GAZE PERCEPTION AND JOINT ATTENTION

\section{References}

Bayliss, A. P., Frischen, A., Fenske, M. J., \& Tipper, S. P. (2007). Affective evaluations of objects are influenced by observed gaze direction and emotional expression. Cognition, 104(3), 644-653.

Bayliss, A. P., Murphy, E., Naughtin, C. K., Kritikos, A., Schilbach, L., \& Becker, S. I. (2013). 'Gaze leading': Initiating simulated joint attention influences eye movements and choice behavior. Journal of Experimental Psychology: General, 142(1), 76-92. doi: $76.10 .1037 / \mathrm{a} 0029286$.

Behrmann, M., \& Tipper, S. P. (1999). Attention accesses multiple reference frames: evidence from visual neglect. Journal of Experimental Psychology: Human Perception and Performance, 25(1), 83.

Böckler, A., van der Wel, R. P. R. D., \& Welsh, T. N. (2014). Catching eyes: Effects of social and nonsocial cues on attention capture. Psychological Science, 25, 720-727. doi: $10.1177 / 0956797613516147$.

Cañadas, E., \& Lupiáñez, J. (2012). Spatial interference between gaze direction and gaze location: A study on the eye contact effect. The Quarterly Journal of Experimental Psychology, 65(8), 1586-1598.

Chen, Y. C., \& Scholl, B. J. (2014). Seeing and liking: Biased perception of ambiguous figures consistent with the "inward bias" in aesthetic preferences. Psychonomic bulletin \& review, 21(6), 1444-1451.

Chen, Y. C., Colombatto, C., \& Scholl, B. J. (2018). Looking into the future: An inward bias in aesthetic experience driven only by gaze cues. Cognition, 176, 209-214.

Edwards, S. G., Stephenson, L., Dalmaso, M., \& Bayliss, A. P. (2015). Social orienting in gaze leading: A mechanism for shared attention. Proceedings of the Royal Society: B, 282(1812). DOI: 10.1098/rspb.2015.1141 
Emery, N. J. (2000). The eyes have it: The neuroethology, function and evolution of social gaze. Neuroscience \& Biobehavioral Reviews, 24, 581-604.

Farah, M. J., Brunn, J. L., Wong, A. B., Wallace, M. A., \& Carpenter, P. A. (1990). Frames of reference for allocating attention to space: Evidence from the neglect syndrome. Neuropsychologia, 28(4), 335-347.

Friesen, C. K., \& Kingstone, A. (2003). Abrupt onsets and gaze direction cues trigger independent reflexive attentional effects. Cognition, 87(1), B1-B10.

Frischen, A., Bayliss, A. P., \& Tipper, S. P. (2007). Gaze cueing of attention: Visual attention, social cognition, and individual differences. Psychological Bulletin, 133, 694-724. doi:10.1037/0033-2909.133.4.694.

Hamilton, A. F. D. C. (2016). Gazing at me: the importance of social meaning in understanding direct-gaze cues. Phil. Trans. R. Soc. B, 371(1686), 20150080.

Hietanen, J. K., Leppänen, J. M., Peltola, M. J., Linna-aho, K., \& Ruuhiala, H. J. (2008). Seeing direct and averted gaze activates the approach-avoidance motivational brain systems. Neuropsychologia, 46(9), 2423-2430.

Jenkins, R., Beaver, J. D., \& Calder, A. J. (2006). I thought you were looking at me: Direction-specific aftereffects in gaze perception. Psychological Science, 17(6), 506513.

Jones, S. (2015). The mediating effects of facial expression on spatial interference between gaze direction and gaze location. The Journal of general psychology, 142(2), 106-117.

Loftus, G. R., \& Masson, M. E. J. (1994). Using confidence intervals in within-subject designs. Psychonomic Bulletin \& Review, 1, 476-490.

Mareschal, I., Calder, A. J., \& Clifford, C. W. (2013). Humans have an expectation that gaze is directed toward them. Current Biology, 23(8), 717-721. 
Marotta, A., Román-Caballero, R., \& Lupiáñez, J. (2018). Arrows don’t look at you: Qualitatively different attentional mechanisms triggered by gaze and arrows. Psychonomic bulletin \& review, 1-6.

Moore, C., \& Dunham, P. (Eds.). (1995). Joint attention: Its origins and role in development. Hillsdale, NJ: Erlbaum.

Nummenmaa, L., \& Calder, A. J. (2009). Neural mechanisms of social attention. Trends in Cognitive Science, 13, 135-143.

Perrett, D. I., Hietanen, J. K., Oram, M. W., Benson, P. J., \& Rolls, E. T. (1992). Organization and functions of cells responsive to faces in the temporal cortex [and discussion]. Philosophical Transactions of the Royal Society B: Biological Sciences, 335 (1273), 23-30.

Reber, R., Winkielman, P., \& Schwarz, N. (1998). Effects of perceptual fluency on affective judgments. Psychological science, 9(1), 45-48.

Redcay, E., Kleiner, M., \& Saxe, R. (2012). Look at this: the neural correlates of initiating and responding to bids for joint attention. Frontiers in human neuroscience, 6, 169.

Schilbach, L., Eickhoff, S. B., Cieslik, E., Shah, N. J., Fink, G. R., \& Vogeley, K. (2010). Eyes on me: an fMRI study of the effects of social gaze on action control. Social cognitive and affective neuroscience, 6(4), 393-403.

Senju, A., \& Hasegawa, T. (2005). Direct gaze captures visuospatial attention. Visual cognition, 12(1), 127-144.

Simmons, J. P., Nelson, L. D., \& Simonsohn, U. (2012). A 21 word solution. Retrieved from $\underline{\text { http://ssrn.com/abstract }=2160588}$

Torres-Marín, J., Carretero-Dios, H., Acosta, A., \& Lupiáñez, J. (2017). Eye contact and fear of being laughed at in a gaze discrimination task. Frontiers in psychology, 8, 1954. 
Tottenham, N., Tanaka, J. W., Leon, A. C., McCarry, T., Nurse, M., Hare, T. A., ... \& Nelson, C. (2009). The NimStim set of facial expressions: judgments from untrained research participants. Psychiatry research, 168(3), 242-249. 